\title{
ON THE JOINS OF SEMIGROUP VARIETIES WITH THE VARIETY OF COMMUTATIVE SEMIGROUPS
}

\author{
M. V. SAPIR AND M. V. VOLKOV
}

(Communicated by Ronald M. Solomon)

\begin{abstract}
We show that the join of a variety of semigroups and the variety of all commutative semigroups is not finitely based, provided some weak conditions.
\end{abstract}

Recall that the join of two varieties $U$ and $V$ is the minimal variety containing $U$ and $V$. To obtain this variety one has to take all homomorphic images of subalgebras of direct products $U \times V$ where $U \in \mathscr{U}, V \in \mathscr{V}$. In [1] it was shown that joins of semigroup varieties with the variety of commutative semigroups Comm very often contain nonfinitely based subvarieties. Here we will show that these joins themselves are often nonfinitely based.

To formulate our result we need one notation. Let $A$ stand for the wellknown completely 0 -simple semigroup over the one-element group $E=\{e\}$ with sandwich matrix $\left[\begin{array}{ll}e & e \\ 0 & e\end{array}\right]$.

Theorem. The join $\mathbf{C o m m}+\mathbf{V}$ has no identity basis with a finite number of variables whenever either $\mathbf{V}$ contains $A$ or $\mathbf{V}$ contains all bands and is locally finite.

Corollary. Let $\mathbf{B}_{m, n}$ be the Burnside variety given by the identity $x^{m}=x^{m+n}$. Then $\mathbf{B}_{m, n}+\mathbf{C o m m}$ has no identity basis with a finite number of variables whenever either $m>1$, or $\mathbf{B}_{m, n}$ is locally finite.

For $m=n=1$ this corollary gives us the result of Nordahl [2]: the join of the variety of bands and the variety of commutative semigroups is not finitely based. This is to say that this result served as an impulse to formulate the above theorem. In fact, we believe that $\mathbf{B}_{m, n}+$ Comm is always nonfinitely based, but we are not able to prove it so far.

Proof of the theorem. Let us suppose first that $\mathbf{V}$ is a periodic variety containing $A$. For every integer $k \geq 3$ we consider the Rees matrix semigroup $T_{k}$ over

Received by the editors May 5, 1992.

1991 Mathematics Subject Classification. Primary 20M07, 20 M14.

The first author was partially supported by NSF grant DMS \#9203981 and by the Center for Communication and Information Sciences of the University of Nebraska at Lincoln.

The second author was partially supported by a grant of the Ministry of Science, Higher School and Technical Policy of Russia, \#2-11-10-44. 
the infinite cyclic group $G=\langle g\rangle$ with the sandwich $(k \times k)$-matrix $P_{k}$

$$
\left[\begin{array}{ccccccc}
g & e & 0 & 0 & \ldots & 0 & 0 \\
0 & e & e & 0 & \ldots & 0 & 0 \\
0 & 0 & e & e & \ldots & 0 & 0 \\
\cdots & \cdots & \ldots & \ldots & \ldots & \cdots & \ldots \\
0 & 0 & 0 & 0 & \ldots & e & e \\
e & 0 & 0 & 0 & \ldots & 0 & e
\end{array}\right],
$$

where $e$ denotes the identity element of $G$. (It should be mentioned that this construction was used first by Mashevitskii in [3].) We are going to prove that $T_{k}$ does not belong to $\mathbf{C o m m}+\mathbf{V}$, but each $(k-1)$-generated subsemigroup of $T_{k}$ belongs to this join. Since $V$ consists of periodic semigroups, it satisfies the identity $x^{m}=x^{m+n}$ for some positive integers $m$ and $n$. Consider the identity

$$
\left(x_{1}^{2} \cdots x_{k}^{2}\right)^{m+n}\left(x_{1} \cdots x_{k}\right)^{m}=\left(x_{1}^{2} \cdots x_{k}^{2}\right)^{m}\left(x_{1} \cdots x_{k}\right)^{m+2 n} .
$$

This identity follows both from the commutativity and from the identity $x^{m}=x^{m+n}$, and therefore it holds in the variety Comm $+\mathbf{V}$. Now we shall show that (1) fails in $T_{k}$. Indeed, substituting the matrix $[e ; i, i] \in T_{k}$ for $x_{i}$, we calculate easily that the left part of $(1)$ is equal to $\left[g^{m+n} ; 1, k\right]$ but the right part of (1) equals $\left[g^{m} ; 1, k\right]$. Since $g^{m+n} \neq g^{m}$ in $G, T_{k}$ does not satisfy (1) and, therefore, does not belong to Comm $+\mathbf{V}$.

For $\alpha=1, \ldots, k$ consider the subsemigroup $T_{k}^{\alpha}$ of $T_{k}$ consisting of 0 and all matrices $[h ; i, j], h \in G$, where $j \neq \alpha$. It is clear that each $(k-1)$ generated subsemigroup of $T_{k}$ is contained in $T_{k}^{\alpha}$ for some $\alpha$. Thus it remains to prove that $T_{k}^{\alpha}$ belongs to Comm $+\mathbf{V}$ for every $\alpha$. We can identify $T_{k}^{\alpha}$ with the Rees matrix semigroup over $G$ with the sandwich matrix $P_{k}^{\alpha}$ obtained from $P_{k}$ by deleting its $\alpha$ th row. Let us multiply in succession the first row of $P_{k}^{\alpha}$ by $g^{-1}$, then the second column of the result by $g$, then the second row by $g^{-1}, \ldots$, and the $(\alpha-1)$ th row by $g^{-1}$, and finally the $\alpha$ th column by $g$. We obtain the matrix $Q_{k}$ consisting of 0 and $e$ only. It is well known (see [4, $\S 3.2])$ that the above-described transformation of the matrix does not change the semigroup $T_{k}^{\alpha}$; in other words, $T_{k}^{\alpha}$ is isomorphic to the Rees matrix semigroup $R_{k}$ over $G$ with sandwich matrix $Q_{k}$. Let $U_{k}$ be the Rees matrix semigroup over the trivial group $E$ with sandwich matrix $Q_{k}$. It is easy to check that the mapping $\pi$ :

$$
\left\{\begin{array}{l}
G \times U_{k} \stackrel{\pi}{\rightarrow} P_{k}, \\
(h,[e ; i, j]) \mapsto[h ; i, j], \\
(h, 0) \mapsto 0,
\end{array}\right.
$$

for all $h \in G, i, j=1, \ldots, k, i \neq \alpha$, is a homomorphism onto $P_{k}$. Now we note that $G \in \mathrm{Comm}$ and any Rees matrix semigroup over $E$ belongs to the variety generated by $A$ (see $[5, \S 20 \mathrm{e}])$. This yields $T_{k}^{\alpha} \cong R_{k} \in \mathbf{C o m m}+\mathbf{V}$.

Let us suppose now that $\mathbf{C o m m}+\mathbf{V}$ has an identity basis involving $r$ variables. Then a semigroup $S$ belongs to $\mathbf{C o m m}+\mathbf{V}$ whenever all its $r$-generated subsemigroups are in Comm $+\mathbf{V}$. Taking $k \geq r$ and $T_{k}$ as $S$, we come to a contradiction.

Now let us suppose that $\mathbf{V}$ is locally finite and contains all bands. We shall use the following result from [6]. Recall that Zimin words $Z_{n}$ are defined as $Z_{1}=x_{1}, \ldots, Z_{n}=Z_{n-1} x_{n} Z_{n-1}$. 
Proposition. If $\mathbf{V}$ is a semigroup variety, all semigroups of $\mathbf{V} \cap \operatorname{var}\left\{x^{2}=0\right\}$ are locally finite, and $\mathbf{V}$ does not satisfy any nontrivial identity of the type $Z_{n}=W$, then $\mathbf{V}$ has no identity basis involving a finite number of variables.

To use this result we have to show that all semigroups in Comm $+\mathbf{V}$ with the additional identity $x^{2}=0$ are locally finite and that $\mathbf{C o m m}+\mathbf{V}$ does not satisfy an identity of the form $Z_{n}=W$.

Let $S$ be a finitely generated semigroup in $\mathbf{C o m m}+\mathbf{V}$ satisfying the identity $x^{2}=0$. As a member of the join $\mathbf{C o m m}+\mathbf{V}, S$ is a factor of an appropriate direct product $G \times A$, where $G$ is a finitely generated semigroup in $\mathbf{V}$ and $A$ is a finitely generated commutative semigroup. Since $\mathbf{V}$ is locally finite, $G$ is finite. Thus $S$ is a homomorphic image of some subsemigroup $R$ of $G \times A$. Since $S$ satisfies $x^{2}=0$, it is an image of the factor semigroup of $R$ over the ideal $I$ generated by all squares. So we may suppose $S \cong R / I$. Let $\sigma$ be the corresponding congruence on $R$, and let $\gamma$ be the congruence on $R$ corresponding to the homomorphism of $R$ into $G$. Denote $R / \sigma \cap \gamma$ by $T$, and denote $I / \gamma$ by $J . J$ is an ideal in $T$, and $T / J \cong S$. Since $J$ is finite $(\gamma$ has a finite index) and squares of all elements of $T$ are in $J, T$ is a periodic semigroup. Clearly, $\gamma$ induces a congruence $\delta$ on $T$ such that $T / \delta \cong R / \gamma$. Every $\delta$-class which is a subsemigroup is a homomorphic image of some $\gamma$-class which is a subsemigroup also. But every such $\gamma$-class is commutative, since $R$ is a subdirect product of finite $G$ and commutative $A$. Thus every $\gamma$-class which is a subsemigroup is periodic and commutative, so it is locally finite. Therefore, $T$ has a congruence $(\delta)$ of finite index such that all classes of $\delta$ which are subsemigroups are locally finite. Hence we can apply a theorem of Brown [7] and conclude that $T$ is locally finite itself. This implies $S$ is locally finite too. Thus every semigroup in Comm $+\mathbf{V}$ satisfying $x^{2}=0$ is locally finite.

It remains to prove that $\mathbf{C o m m}+\mathbf{V}$ does not satisfy any nontrivial identity of type $Z_{n}=W$. We shall prove that even the join of the variety of bands and the variety of all commutative semigroups cannot satisfy such an identity. Suppose this join satisfies identity $Z_{n}=W$ for some $n$ and $W$. Recall that by the definition $Z_{n}=Z_{n-1} x_{n} Z_{n-1}$, so $x_{n}$ occurs in $Z_{n}$ only one time. Since the identity $Z_{n}=W$ holds in the variety of all commutative semigroups, it is a balanced identity. It means that every letter occurs the same number of times in its left and right parts. For instance, $x_{n}$ must occur in $W$ exactly one time. Hence $W$ can be represented in the form $W_{0} x_{n} W_{1}$ for some words $W_{0}$ and $W_{1}$ which do not contain $x_{n}$. If $U$ is a word, as usual [8], let $\gamma(U)$ $(\delta(U))$ stand for the minimal left (right) part of $U$ containing all variables occurring in $U$. If $x$ is a variable, then $l_{x}(U)$ stands for the number of occurrences of $x$ in $U$. For example, $\gamma\left(Z_{n}\right)=\delta\left(Z_{n}\right)=Z_{n-1}, l_{x_{n}}\left(Z_{n}\right)=1$. As is well known [8], if an identity $U=V$ holds in the variety of all bands, then identities $\gamma(U)=\gamma(V)$ and $\delta(U)=\delta(V)$ also hold in this variety. Hence, it must satisfy identities $\gamma\left(Z_{n}\right)=\gamma(W)$ and $\delta\left(Z_{n}\right)=\delta(W)$, but it is easy to see that $\gamma(W)=W_{0}$ and $\delta(W)=W_{1}$. The first one of these identities has the form $Z_{n-1}=W_{0}$, and the second one has the form $Z_{n-1}=W_{1}$. Clearly, one of these identities is nontrivial. If one of them is trivial, then the other is balanced, and we get the contradiction with the minimality of $n$. Assume both identities are nontrivial. Hence there is a number $k<n$ such 
that numbers $l_{x_{k}}\left(Z_{n-1}\right), l_{x_{k}}\left(W_{0}\right)$, and $l_{x_{k}}\left(W_{1}\right)$ are different. Take the maximal $k$ satisfying this property. Without loss of generality we may suppose that $l_{x_{k}}\left(Z_{n-1}\right)>l_{x_{k}}\left(W_{1}\right)$, because $l_{x_{k}}\left(W_{0}\right)+l_{x_{k}}\left(W_{1}\right)=2 l_{x_{k}}\left(Z_{n-1}\right)$. If $k=n-1$ then we have $1=l_{x_{k}}\left(Z_{n-1}\right)>l_{x_{k}}\left(W_{1}\right)$ whence $l_{x_{k}}\left(W_{1}\right)=0$. Therefore, in this case the identity $Z_{n-1}=W_{1}$ is heterogeneous, and the variety of all bands cannot satisfy it (in fact, even the variety of all semilattices cannot satisfy such an identity), so $k<n-1$. We may suppose that the identity $Z_{n}=W$ was chosen in such a way that the difference between the number of variables in this identity and $k$ is minimal. Again we have $Z_{n-1}=Z_{n-2} x_{n-1} Z_{n-2}, W_{1}=W_{01} x_{n-2} W_{11}$; words $Z_{n-2}, W_{01}, W_{11}$ do not contain $x_{n-2}, \gamma\left(Z_{n-1}\right)=\delta\left(Z_{n-1}\right)=Z_{n-2}$, $\gamma\left(W_{1}\right)=W_{01}, \delta\left(W_{1}\right)=W_{11}$; and the variety of all bands satisfies identities $Z_{n-2}=W_{01}$ and $Z_{n-2}=W_{11}$. Of course, either $l_{x_{k}}\left(Z_{n-2}\right)>l_{x_{k}}\left(W_{01}\right)$ or $l_{x_{k}}\left(Z_{n-2}\right)>l_{x_{k}}\left(W_{01}\right)$. Let, say, the first possibility hold. It is easy to see that the difference between the number of variables in this identity and $k$ is less than that for the identity $Z_{n-1}=W_{1}$, so we get a contradiction. This contradiction completes the proof of the theorem.

\section{REFERENCES}

1. M. V. Volkov and M. V. Sapir, $H F B$ property and structure of semigroups, Contrib. Gen. Algebra, vol. 6, 1988, pp. 303-310.

2. T. Nordahl, On the join of the variety of all bands and the variety of all commutative semigroups via conditional rewrite rules, Proceedings of the International Colloquium on Words, Semigroups and Combinatorics, Kioto, 1990.

3. G. I. Mashevitskii, An example of a finite semigroup without irreducible identity basis in the class of completely 0-simple semigroups, Uspekhi Mat. Nauk 38 (1983), 211-213. (Russian)

4. A. H. Clifford and G. B. Preston, The algebraic theory of semigroups, vol. 1, Amer. Math. Soc., Providence, RI, 1961.

5. L. N. Shevrin and M. V. Volkov, Identities of semigroups, Izv. Vyssh. Uchebn. Zaved. Mat. 1985, no. 11, 3-47. (Russian)

6. M. V. Sapir, Burnside type problems and finite basis property in semigroup varieties, Izv. Akad. Nauk SSSR Ser. Mat. 51 (1987), 319-340. (Russian)

7. T. C. Brown, On locally finite semigroups, Ukrain. Mat. Zh. 20 (1968), 732-738. (Russian)

8. C. F. Fennemore, All varieties of bands, Math. Nachr. 48 (1971), 237-262.

Department of Mathematics and Statistics, Center for Information and CommuniCATION SCIENCES, University OF Nebraska, LinColn, Nebraska 68506

E-mail address: sapir@hoss.unl.edu

Department of Mathematics and Mechanics, Urals State University, Lenina 51, EKaTERINBURG 620083, RUSSIAN Federation

E-mail address: volkovm@urgu.e-burg.su 